\title{
Interplay between enterobactin, myeloperoxidase and lipocalin 2 regulates $E$. coli survival in the inflamed gut
}

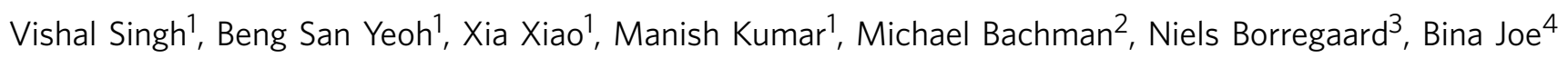
\& Matam Vijay-Kumar ${ }^{1,5}$

During an inflammatory response in the gut, some commensal bacteria such as $E$. coli can thrive and contribute to disease. Here we demonstrate that enterobactin (Ent), a catecholate siderophore released by $E$. coli, is a potent inhibitor of myeloperoxidase (MPO), a bactericidal enzyme of the host. Glycosylated Ent (salmochelin) and non-catecholate siderophores (yersiniabactin and ferrichrome) fail to inhibit MPO activity. An E. coli mutant $(\triangle f e p A)$ that overproduces Ent, but not an Ent-deficient double mutant ( $\triangle a r o B / \Delta f e p A)$, inhibits MPO activity and exhibits enhanced survival in inflamed guts. This survival advantage is counter-regulated by lipocalin 2, a siderophore-binding host protein, which rescues MPO from Ent-mediated inhibition. Spectral analysis reveals that Ent interferes with compound I [oxoiron, $\mathrm{Fe}(\mathrm{IV})=\mathrm{O}]$ and reverts the enzyme back to its native ferric [Fe(III)] state. These findings define a fundamental mechanism by which $E$. coli surpasses the host innate immune responses during inflammatory gut diseases and gains a distinct survival advantage.

\footnotetext{
${ }^{1}$ Department of Nutritional Sciences, Pennsylvania State University, University Park, Pennsylvania 16802, USA. ${ }^{2}$ Department of Pathology, University of Michigan, Ann Arbor, Michigan 48109-5602, USA. ${ }^{3}$ Department of Hematology, The Granulocyte Research Laboratory, National University Hospital, University of Copenhagen, Copenhagen 2100, Denmark. ${ }^{4}$ Department of Physiology and Pharmacology, Center for Hypertension and Personalized Medicine, The University of Toledo College of Medicine and Life Sciences, Toledo, Ohio 43614, USA. 5 Department of Medicine, Pennsylvania State University Medical Center, Hershey, Pennsylvania 17033, USA. Correspondence and requests for materials should be addressed to M.V.-K. (email: mvk13@psu.edu).
} 
T he mammalian intestine harbours a community of bacteria collectively known as the microbiota. Both the host and the microbiota depend on and compete for common nutrients, such as the essential trace transition metal iron, for their proliferation and biologic functions. In the host, the majority of circulating iron is bound to host proteins, resulting in low free-iron availability $\left(10^{-24} \mathrm{M}\right)$, which functions as a 'natural antibiotic/nutritional immunity' mechanism for controlling bacterial growth ${ }^{1-3}$. The bacteria circumvent low iron levels in the host by expressing a suite of siderophores with high affinity for iron. Enterobactin (Ent), a prototypical and conserved siderophore, has an unmatched affinity for iron $\left(K_{\mathrm{d}} \text { of } 10^{-35} \mathrm{M} \text { at physiologic } \mathrm{pH}\right)^{4}$, allowing Ent to chelate iron from iron-binding host proteins such as ferritin and transferrin. The monomeric form of Ent, designated 2,3-dihydroxybenzoic acid (DHBA), is produced by Brucella abortus under conditions of low iron availability ${ }^{5}$. Previous studies have demonstrated that pathogens that synthesize defective siderophores are less virulent ${ }^{6,7}$. In fact, many pathogens sense the low iron levels in the host (for example, hypoferremia of inflammation) as an environmental cue to trigger synthesis of virulence factors ${ }^{3,8}$. Further, iron acquisition by siderophores plays a significant role in extraintestinal pathogenic E. coli virulence? .

Neutrophils are the most abundant white blood cells in circulation and are the first responder cell type during infection, inflammation and injury. Myeloperoxidase (MPO, Enzyme Commission: 1.11.2.2), a haem protein and a marker of neutrophils, requires $\mathrm{Fe}^{+3}$ for its bioactivity. During respiratory burst, MPO uses $\mathrm{H}_{2} \mathrm{O}_{2}$ and halide ions to generate hypochlorous acid, a potent oxidizing and antimicrobial agent ${ }^{10}$. Lactoperoxidase (LPO, Enzyme Commission: 1.11.1.7), a similarly haemcontaining milk protein, shares a number of overlapping properties with $\mathrm{MPO}^{11}$. In neutrophils, MPO is a companion of the siderophore-binding protein lipocalin 2 ( $\mathrm{Lcn} 2$, also known as neutrophil gelatinase-associated lipocalin). Lcn2 acts as part of the first line of defence against invading microbes by chelating bacterial siderophores with high affinity $(0.4 \mathrm{nM})^{1}$, depriving them of iron. Although Lcn2 has no measurable affinity for $\mathrm{Fe}^{+3}$ alone, it can bind to siderophores alone or to $\mathrm{Fe}^{+3}$-bound siderophores (for example, Ent or $\mathrm{Fe}^{+3}$-Ent) ${ }^{12}$.

Gut bacterial dysbiosis is associated with a number of gastrointestinal disorders including human immunodeficiency virus enteropathy ${ }^{13}$, necrotizing enterocolitis ${ }^{14}$, Crohn's disease ${ }^{15}$, irritable bowel syndrome ${ }^{16}$, inflammation-associated enterotype ${ }^{17}$, antibiotic treatment ${ }^{18}$, enteropathogen infection, chemical- and genetically induced colitis ${ }^{19}$ and protozoan parasite infection ${ }^{20}$. One of the potential mechanisms by which Enterobacteriaceae flourish despite the inflammatory milieu is through utilization of stable reactive oxygen and nitrogen species produced as by-products of the host innate inflammatory response as electron acceptors, therefore supporting their growth and anaerobic respiration ${ }^{21-23}$.

In this study, we show that the bacterial siderophore Ent and its monomeric form DHBA inhibit MPO/LPO activity in vitro and inhibit MPO activity in the inflamed gut. Inhibition of MPO activity by Ent confers a distinct survival advantage and permits enhanced colonization by $E$. coli in the inflammatory state. Elucidation of possible underlying mechanisms via spectral analysis using $\mathrm{LPO}-\mathrm{H}_{2} \mathrm{O}_{2}$ as a model system reveals that Ent acts as a potent, fast-acting suicide substrate that prevents hypohalous acid formation in the peroxidase-catalysed reaction. Collectively, our study describes a novel mechanism that promotes survival and growth of E. coli, a well-known opportunistic pathogen in a variety of chronic intestinal diseases associated with microbiotal dysbiosis.

\section{Results}

Apo-enterobactin is a potent inhibitor of MPO activity. Microbiotal dysbiosis is commonly observed in several types of inflammatory bowel diseases (IBDs). Specifically, IBD is associated with a bloom in bacterial species belonging to the family Enterobacteriaceae (for example, E. coli), class Gammaproteobacteria, within the phylum proteobacteria. It has been shown that $E$. coli thrives in inflammatory conditions by expressing several genes that confer a survival advantage. In this study, we asked whether the $\mathrm{Fe}^{+3}$-chelating siderophore, apo-Ent $\left(\mathrm{Fe}^{+3}\right.$ free), could affect the bioactivity of the dimeric haem protein MPO, which requires $\mathrm{Fe}^{+3}$ for its function. As shown in Fig. 1a, cyclic apo-Ent inhibited MPO activity in a dose-dependent manner. Specifically, apo-Ent at 1.0 and $30 \mu \mathrm{M}$ concentration inhibited 50 and $80 \%$ of MPO activity, respectively. DHBA, an intermediate in the Ent biosynthesis with lower affinity for $\mathrm{Fe}^{+3}$ than Ent, also inhibited MPO activity in a dose-dependent manner, although to a lesser extent than Ent. The dose-response curve and time-kinetic response of MPO inhibition by Ent and DHBA revealed that Ent is a more potent and fast-acting inhibitor of MPO activity than DHBA (Fig. 1a,b). Nevertheless, both Ent and DHBA are more potent and fast-acting inhibitors of MPO activity than 4-aminobenzoic hydrazide $(\mathrm{ABAH})$, a known inhibitor of $\mathrm{MPO}^{24}$. In a similar fashion, Ent and DHBA inhibited monomeric haem protein LPO activity in a dose- and time-dependent manner (Supplementary Fig. 1). Surprisingly, DHBA was found to be more potent than Ent in inhibiting LPO activity (Supplementary Fig. 1A). The inhibitory effect of Ent on MPO activity was observed even after the initiation of the MPO reaction and over a range of temperatures and $\mathrm{pH}$ (Supplementary Fig. 2A-C). To determine whether inhibition of MPO or LPO is an inherent property of all siderophores or is specific to Ent, we tested MPO and LPO inhibitory activity of salmochelin, yersiniabactin and ferrichrome, which showed only $\sim 13, \sim 5$ and $\sim 0.5 \% \mathrm{MPO}$ inhibitory activity, respectively, even at concentrations 50 times greater than Ent (Supplementary Fig. 3A-C). In the same way, these siderophores were weak inhibitors of LPO activity (Supplementary Fig. 3D-F). Taken together, these observations suggest that MPO and LPO inhibitory activity is relatively more specific to Ent.

During conditions of iron scarcity, bacteria drastically upregulate expression of siderophores to acquire this essential metal. After binding $\mathrm{Fe}^{+3}$ in the stoichiometry of $1: 1$, Ent is internalized by bacteria via the FepA receptor. Next, we asked whether $\mathrm{Fe}^{+3}$-bound Ent possesses MPO inhibitory activity. We incubated $\mathrm{Fe}^{+3}$ (sourced from $\mathrm{FeCl}_{3}$ ) with apo-Ent in a 1:1 and $3: 1$ ratio at $37^{\circ} \mathrm{C}$ for 5 min. Interestingly, $\mathrm{Fe}^{+3}$-Ent in a ratio of 3:1 completely failed to inhibit MPO activity, whereas $\mathrm{Fe}^{+3}$-Ent in the 1:1 ratio was still able to inhibit MPO activity to some extent (Fig. 1c). $\mathrm{FeCl}_{3}$ alone at $30 \mu \mathrm{M}$ did not influence the MPO-catalysed reaction. Similarly, $\mathrm{Fe}^{+3}$-DHBA (1:1) failed to inhibit MPO activity (Fig. 1c), suggesting that the inhibitory effect of Ent on these iron-containing enzymes require its $\mathrm{Fe}^{+3}$-free form.

To delineate the possible underlying mechanism of Entinduced inhibition of MPO activity, we performed spectral analysis of a peroxidase-catalysed reaction with $\mathrm{H}_{2} \mathrm{O}_{2}$ in the presence or absence of apo forms of Ent and DHBA using LPO. LPO exhibits similar activity to MPO but is more affordable (than MPO) and therefore practical for usage in spectral analyses. Before starting spectral analysis, we monitored the characteristic spectra of LPO, which showed $\lambda_{\max }$ at $412 \mathrm{~nm}$ (Fig. 1d). Upon addition of $\mathrm{H}_{2} \mathrm{O}_{2}(30 \mu \mathrm{M})$, LPO was converted into a redox intermediate with $\lambda_{\max }$ at $\sim 430 \mathrm{~nm}$, which is characteristic of the compound II ( $\mathrm{Fe}(\mathrm{IV})=\mathrm{OH}$-porphyrin) (Fig. 1e). 
a

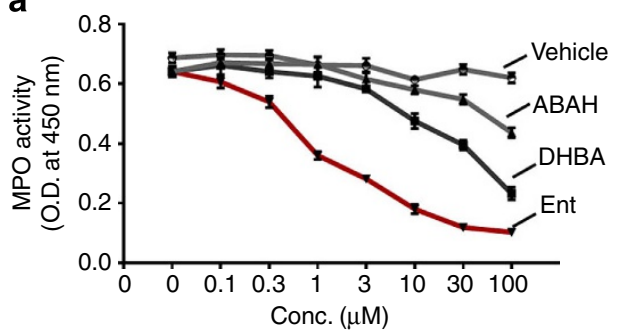

b

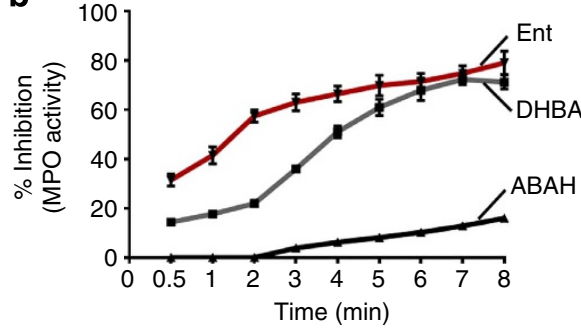

C

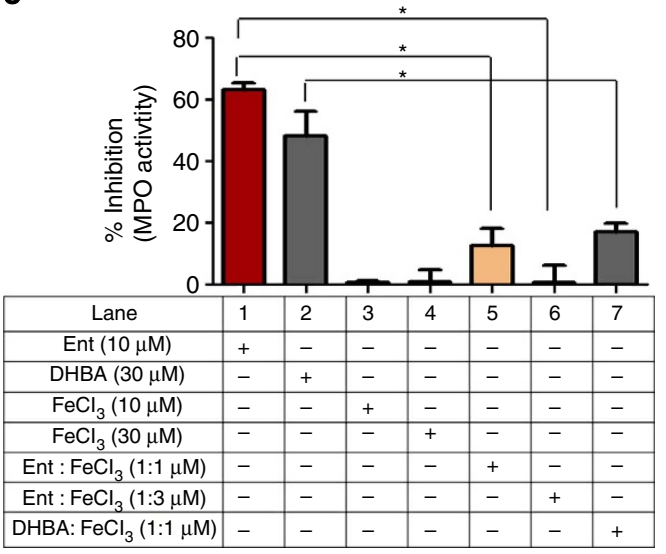

d

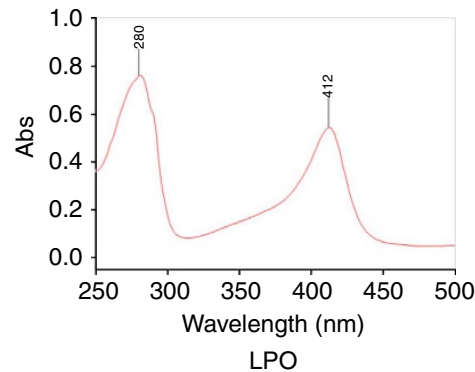

e f

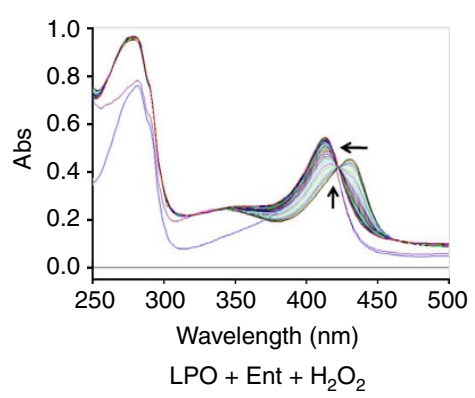

i

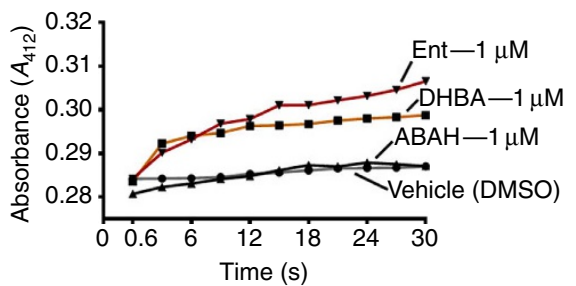

g

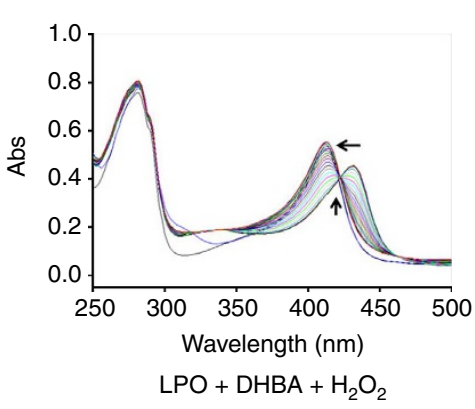

j

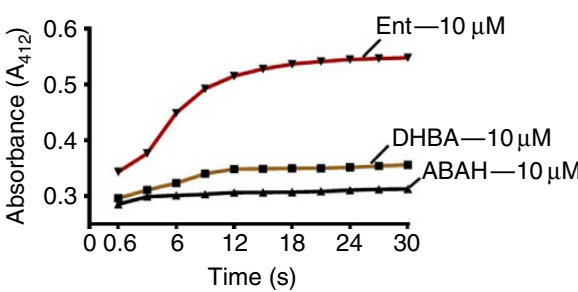

h

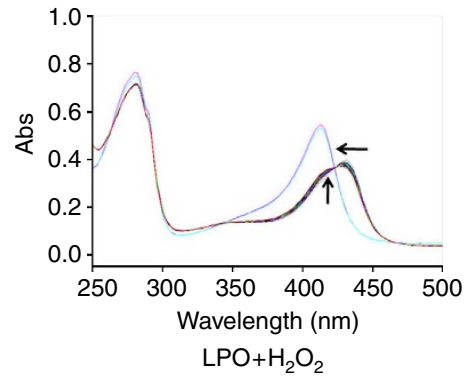

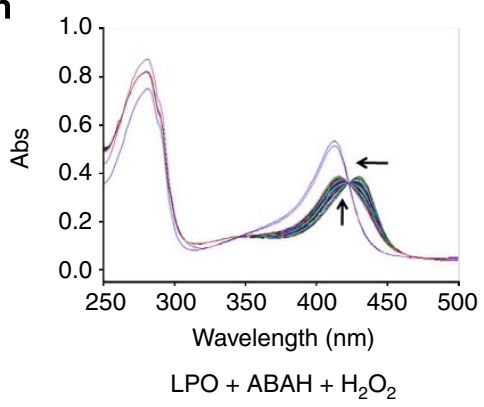

k

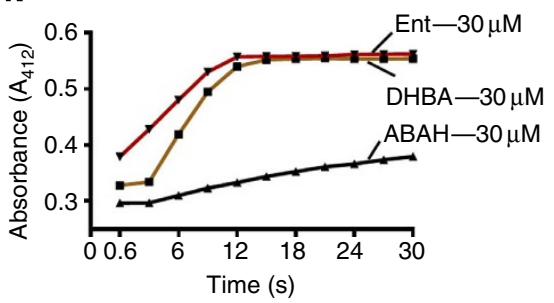

Figure 1 | Aferric $\mathbf{E}$. coli siderophore enterobactin (Ent) inhibits MPO activity. Ent, DHBA or ABAH was pre-incubated at the below indicated final concentrations, with $100 \mathrm{mU}$ of MPO for $10 \mathrm{~min}$ and then MPO activity was quantitatively measured using guaiacol $(100 \mathrm{mM})$ and $\mathrm{H}_{2} \mathrm{O}_{2}\left(6.7 \times 10^{-3} \%\right)$. Line graph represents (a) dose- $(0.1-100 \mu \mathrm{M})$ and (b) time (0.5-8 min)-dependent inhibition of MPO activity by $30 \mu \mathrm{M}$ of Ent, DHBA or ABAH. (c) Ent or DHBA was pre-incubated with $\mathrm{FeCl}_{3}$ for $1 \mathrm{~min}$ and subsequently incubated with MPO for $10 \mathrm{~min}$. Bar graph represents percent inhibition of MPO activity by apo- or $\mathrm{Fe}^{+3}$-saturated Ent or DHBA. Assays were carried out in 96-well plates in triplicate with appropriate vehicles/buffers throughout the study. (d-h) To perform spectral analysis, $\mathrm{LPO}(2.6 \mathrm{nM}$, in $0.1 \mathrm{M}$ phosphate buffer) was incubated with various concentrations of Ent, DHBA or ABAH for 1 min. The reaction was initiated by the addition of $30 \mu \mathrm{M} \mathrm{H}_{2} \mathrm{O}_{2}$. Spectra $(250-500 \mathrm{~nm})$ were recorded up to $3 \mathrm{~min}$. Each spectrum is an average of three scans taken in $1.0 \mathrm{~s}$. Image displays the spectra of (d) LPO alone, (e) LPO $+\mathrm{H}_{2} \mathrm{O}_{2}$, (f) LPO + Ent $(30 \mu M)+\mathrm{H}_{2} \mathrm{O}_{2}$, (g) LPO $+\mathrm{DHBA}(30 \mu M)+\mathrm{H}_{2} \mathrm{O}_{2}$ and (h) LPO $+\mathrm{ABAH}$ $(30 \mu M)+\mathrm{H}_{2} \mathrm{O}_{2}$. (i-k) Time-dependent change in the absorbance spectra of $L P O$ at $412 \mathrm{~nm}$ at various concentrations of ABAH, Ent and DHBA. Arrows indicate the direction of spectral changes, that is, conversion of oxoiron $[\mathrm{Fe}(\mathrm{IV})=\mathrm{O}]$ to the native form $[\mathrm{Fe}(\mathrm{III})]$. Results are expressed as mean \pm s.e.m. and are representative of six independent experiments $(\mathbf{a}-\mathbf{c})$. Data represent one of three similar experiments $(\mathbf{f}-\mathbf{k}){ }^{\star} P<0.05$, unpaired $t$-test.

In the presence of Ent $(30 \mu \mathrm{M}), \mathrm{H}_{2} \mathrm{O}_{2}$ mediated a shift in the Soret band and reverted back (denoted by arrows) to $412 \mathrm{~nm}$ within seconds, suggesting the regeneration of native LPO (Fig. 1f). Similarly, DHBA at $30 \mu \mathrm{M}$ completely reverted the shifted peak to $412 \mathrm{~nm}$ within seconds (Fig. 1g). In agreement with our in vitro data, $\mathrm{ABAH}(30 \mu \mathrm{M})$ did not completely revert the $\mathrm{H}_{2} \mathrm{O}_{2}$-induced shift in LPO spectra (Fig. 1h), suggesting it is a weaker inhibitor than Ent or DHBA (Fig. 1a,b). Furthermore, a 
dose-dependent response of Ent, DHBA and $\mathrm{ABAH}$ on the regeneration of native $\mathrm{LPO}$ revealed that both apo-Ent and DHBA are more potent and fast-acting suicide substrates than ABAH (Fig. 1i-k). The haem [Fe(III)] in LPO participates in a redox reaction, and is converted to oxoiron $[\mathrm{Fe}(\mathrm{IV})=\mathrm{O}$ ], also known as 'compound I', upon the addition of $\mathrm{H}_{2} \mathrm{O}_{2}$. In the presence of a halide ion, compound $\mathrm{I}$ is reduced back to its ferric state, $[\mathrm{Fe}(\mathrm{III})]$ and this results in hypohalous acid formation, a potent bactericidal agent. As we observed in our spectral analyses, Ent and DHBA reduced the oxoiron $[\mathrm{Fe}(\mathrm{IV})=\mathrm{O}]$ to the native enzyme [Fe(III)], effectively preventing the formation of hypohalous acid. There was no change in the spectra of LPO observed upon addition of Ent, DHBA or ABAH in the absence of $\mathrm{H}_{2} \mathrm{O}_{2}$, suggesting that they do not directly interact with $\mathrm{Fe}^{+3}$ in the haem moiety (Supplementary Fig. 4A-D). Collectively, these results demonstrate that both Ent and DHBA are fast-acting suicide substrates that accelerate native peroxide formation and consequently prevent the generation of the potent oxidizing antimicrobial agent hypohalous acid.

E. coli-produced enterobactin inhibits MPO activity. Host inflammatory conditions are generally characterized by hypoferremia $^{25}$, an environmental signal that prompts bacteria to overexpress siderophores ${ }^{3}$. To address whether Ent-expressing bacteria can exert MPO inhibitory activity, we took advantage of
E. coli mutants that either overproduce Ent $(\triangle f e p A)$ or lack Ent production $(\Delta a r o B, \Delta e n t C$ and $\Delta a r o B / \Delta f e p A)$. On the basis of 8-h broth growth curves in triplicate, we did not observe any significant difference in growth of theses mutants in LB medium (Supplementary Fig. 5). E. coli and its isogenic mutants were plated on chrome azurol S (CAS) plates to detect the expression of the siderophore. This assay is founded on the observation that CAS gives a distinct blue colour when in complex with iron. While iron is chelated by siderophores effectively, an orange halo forms around the siderophore-producing bacteria. As expected, $\triangle f e p A$ showed a prominent orange-coloured halo, which was much greater than wild-type (WT) E. coli. Also as predicted, no halo formation was observed for $\triangle a r o B, \Delta e n t C$ (Fig. 2a), or $\triangle a r o B / \Delta f e p A$ mutants (Fig. 3a, upper panel). Overnight-grown $\triangle f e p A\left(10^{6}\right.$ colony-forming units (CFUs) per $\left.\mathrm{ml}\right)$ produced $\sim 2.5 \mu \mathrm{g}$ of Ent (Fig. 2b), as quantified on CAS agar plates. Importantly, ferric-iron-saturated Ent did not form halos in this assay, confirming that the Ent used in this study was the aferric form (Fig. 2c). In addition, the overnight culture supernatants (normalized to equivalent number of bacteria) with or without dipyridyl were tested for MPO inhibitory activity. The irondepleted bacterial culture supernatant obtained from $\Delta f e p A$ showed substantial inhibition of MPO activity when compared with WT E. coli, whereas the supernatants obtained from $\triangle a r o B$ and $\triangle e n t C$ did not exhibit MPO inhibitory activity (Fig. 2d). Interestingly, the inhibitory activity of iron-sufficient LB culture a

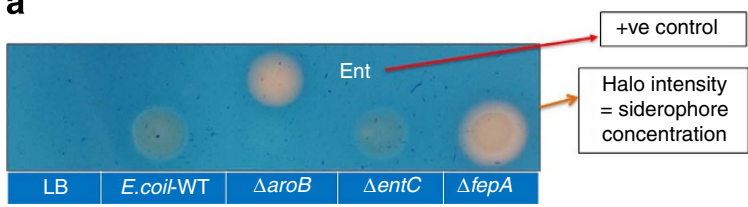

b

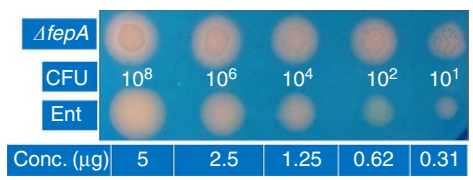

C

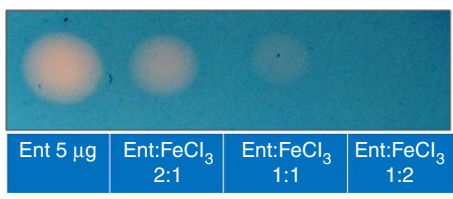

c

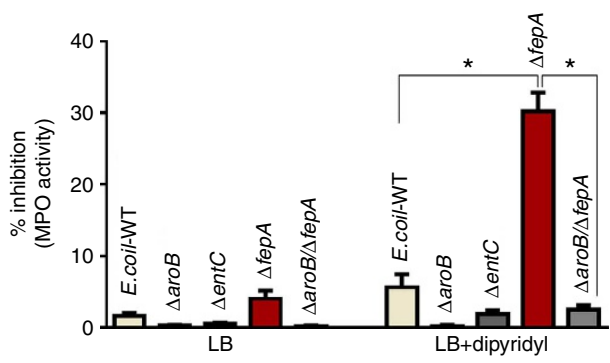

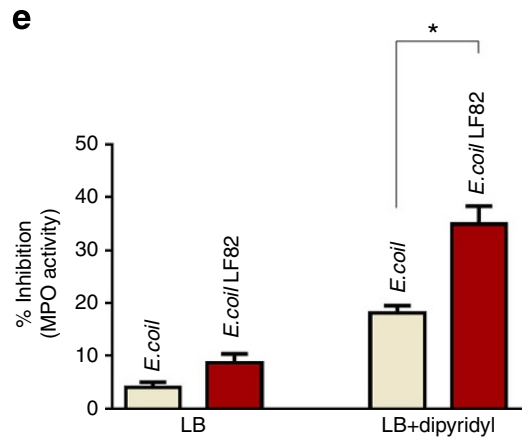

Figure 2 | Ent-overproducing $\boldsymbol{E}$. coli exerts more potent MPO inhibitor activity. WT $E$. coli and its isogenic Ent mutants $\triangle a r o B, \Delta e n t C, \Delta f e p A$ and $\Delta$ aroB/ $\triangle$ fepA were grown overnight in LB and subsequently grown in LB media containing iron chelator 2,2'-dipyridyl ( $350 \mu \mathrm{M})$ for another $6 \mathrm{~h}$. Afterwards, bacteria were pelleted down and supernatants were tested for their MPO inhibitory activity. (a) Representative photograph showing siderophore production on CAS plates by WT E. coli and Ent mutants. (b) Ent-overproducing $\triangle$ fepA mutant was plated on CAS plate. Different concentrations of apo-Ent (Sigma) were applied on the CAS plate to quantitate the amount of Ent produced by $\Delta$ fepA mutant. (c) Ent saturated with $\mathrm{FeCl}_{3}$ in different proportions was plated on the CAS plate. (d) Bar graph displaying the \% inhibition of MPO activity by spent media obtained from WT E. coli or Ent mutants grown in LB or LB $+2,2^{\prime}$-dipyridyl. (e) WT and the adherent-invasive E. coli strain LF82 were grown as in $\mathbf{d}$ and the clear supernatants were used to test their MPO inhibitory activity. Results are expressed as mean \pm s.e.m. and are representative of six independent experiments. ${ }^{\star} P<0.05$, unpaired $t$-test. 
supernatants from WT and fepA mutants was modest (Fig. 2d). This result suggests that Ent or its precursors secreted into the media may be saturated with the $\mathrm{Fe}^{+3}$ present in the LB media, or that the relatively high iron in LB might repress Fur-mediated biosynthesis of siderophores, specifically of Ent. These findings support the above observation (Fig. 1c) that only the apo forms of Ent and DHBA exhibit inhibition of MPO. Similar to $\triangle f e p A$, supernatants from an adherent-invasive $E$. coli strain (LF82) isolated from a Crohn's disease patient ${ }^{26}$ exhibited increased MPO inhibitory activity (Fig. 2e). To test the stability of $\Delta f e p A$ supernatant, we subjected the supernatant to various conditions $\left(24 \mathrm{~h}\right.$ at $-80^{\circ} \mathrm{C}, 4^{\circ} \mathrm{C}$, room temperature $\left(\sim 25^{\circ} \mathrm{C}\right), 37^{\circ} \mathrm{C}$, freeze/ thawed three times or boiled for $5 \mathrm{~min})$. With the exception of boiling, $\triangle f e p A$ supernatant retained its MPO and LPO inhibitory activity after the treatments (Supplementary Fig. 6).

Ent-overproducing $E$. coli inactivates MPO in colitic mice. Next, we performed in vivo studies to determine whether introducing Ent mutants to mice with intestinal inflammation would inhibit MPO activity. We employed infectious Salmonellainduced gastroenteritis and chemical colitogen dextran sulfate sodium (DSS)-induced colitis models, which are characterized by caecal and colonic inflammation, respectively. As shown in our previous studies ${ }^{27,28}$, Salmonella-induced gastroenteritis in mice pretreated with streptomycin exhibited caecal emptying and shrinkage. Mice with caecal inflammation inoculated with WT $E$. coli or its isogenic mutants $\triangle f e p A$ or $\triangle a r o B$ did not show significant alterations in inflammatory cell infiltrate or caecal pathology, as determined with haematoxylin and eosin staining of caecal sections (Supplementary Fig. 7A). Similarly, colonic pathology and infiltration of macrophages were unaltered in mice that received WT $E$. coli or its isogenic mutants $\triangle f e p A$ or $\triangle a r o B / \Delta f e p A$ when compared with control animals (given DSS alone) in the experimental model of DSS-induced acute colitis (Supplementary Fig. 7B,C).

Salmonella-given mice that were inoculated with WT E. coli or $\Delta f e p A$, but not $\triangle a r o B$ or $\Delta a r o B / \Delta f e p A$, demonstrated a consistent reduction in caecal MPO activity when compared with control mice given Salmonella alone (Fig. 3a). Likewise, mice given WT E. coli or $\Delta f e p A$, but not $\Delta a r o B$ or $\Delta a r o B / \Delta f e p A$, presented with a significant reduction in colonic MPO levels in the DSS-induced colitis model (Fig. 3b). Further, orally administered DHBA also substantially reduced MPO activity in all three regions of the colon (proximal, middle and distal) of DSS-treated mice (Fig. 3c). These results indicate that Ent can inhibit MPO activity in colitic mice whether generated in vivo by $E$. coli or administered orally.

Ent provides survival advantage to $E$. coli in the inflamed gut. To evaluate whether Ent-induced inhibition of MPO confers a survival advantage to $E$. coli in vitro by limiting generation of the bactericide hypohalous acid ${ }^{10}$, we measured the viability of WT E. coli exposed to MPO- $\mathrm{H}_{2} \mathrm{O}_{2}$ in the presence or absence of Ent or DHBA. As shown in Fig. 4a,b, Ent and DHBA substantially reduced the bactericidal activity of $\mathrm{MPO}-\mathrm{H}_{2} \mathrm{O}_{2}$. Notably, $\mathrm{Fe}^{+3}$-bound Ent or DHBA (1:1 stoichiometric ratio) failed to prevent MPO- $\mathrm{H}_{2} \mathrm{O}_{2}$-mediated bacterial killing (Fig. 4b), confirming our previous observation that the aferric form of Ent is required for inhibition of MPO activity. Both Ent and DHBA rescued bacteria from $\mathrm{LPO}-\mathrm{H}_{2} \mathrm{O}_{2}$-mediated killing

a
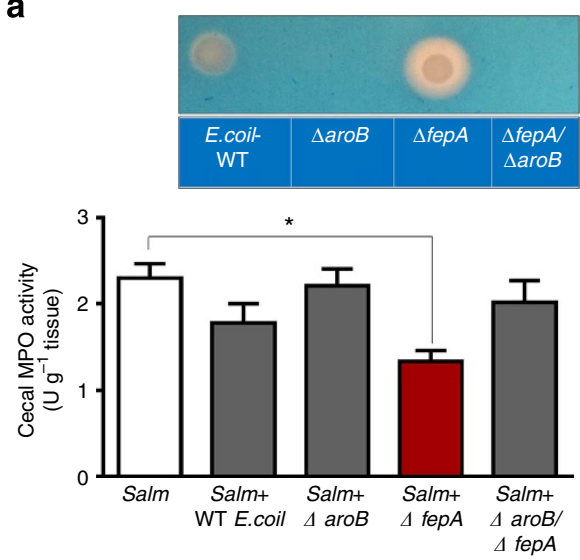

b

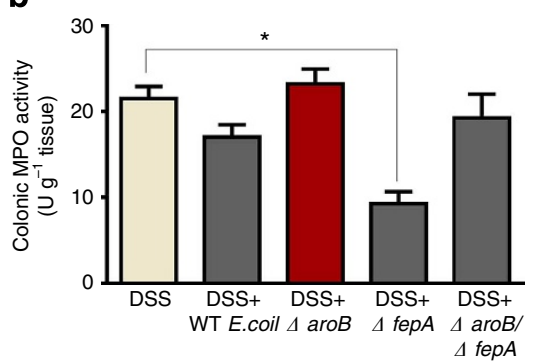

C

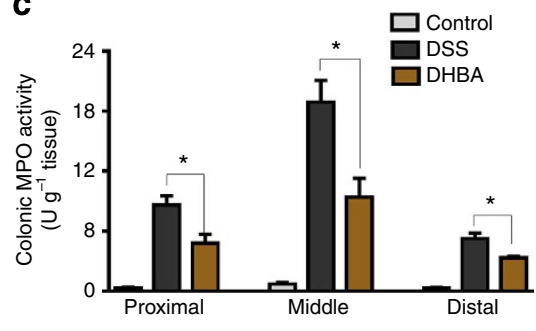

Figure 3 | Inhibition of MPO activity by Ent and DHBA in experimental colitis. Salmonella and DSS-induced colitic mice were orally gavaged with overnight-grown WT E. coli or Ent mutants ( $10^{9} \mathrm{CFU}$ per mouse, $n=4-5$ ). Mice were euthanized via $\mathrm{CO}_{2}$ asphyxiation and caecal and colonic MPO activity was subsequently quantified. (a) Caecal MPO, upper panel showing lack of Ent production in $\Delta a r o B / \Delta f e p A$ mutant. (b) Colonic (middle portion) tissue samples from the DSS-treated group. (c) DSS-induced colitic mice $(n=4)$ were orally gavaged with DHBA ( $100 \mathrm{mg} \mathrm{kg}^{-1}$ body weight) and after $3 \mathrm{~h}$, mice were euthanized via $\mathrm{CO}_{2}$ asphyxiation and MPO activity was quantified in proximal, middle and distal regions of colon. Results are expressed as mean \pm s.e.m. and are representative of two independent experiments with 4-5 mice per group. ${ }^{\star} P<0.05$, unpaired $t$-test. 
a

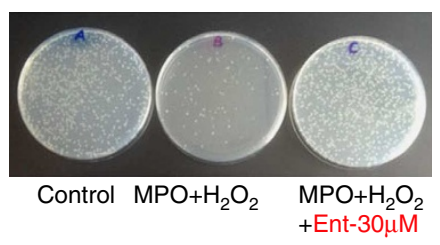

b

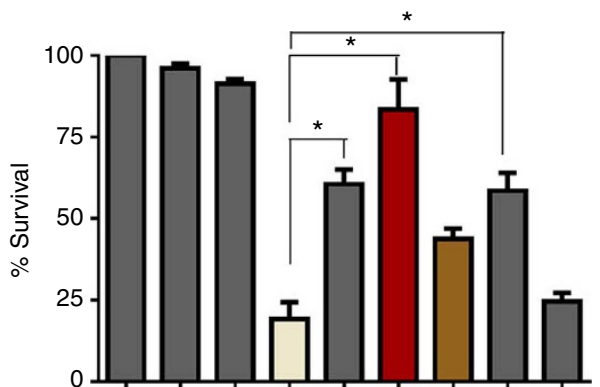

C

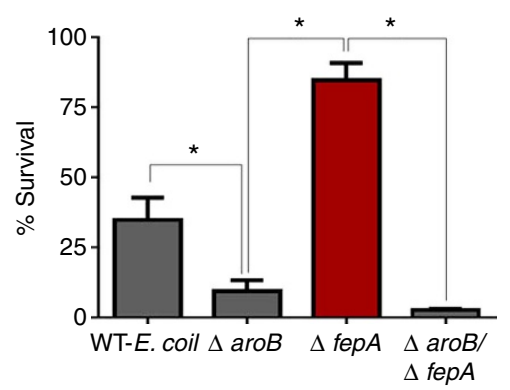

d

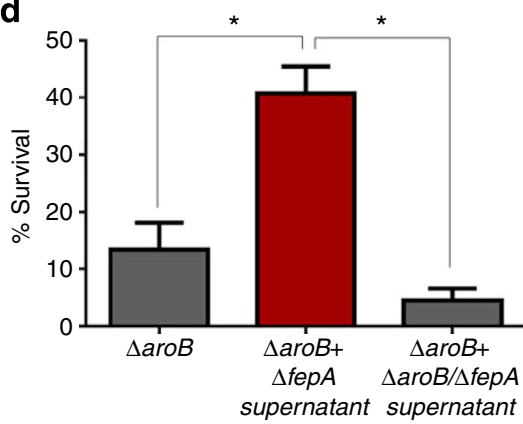

h

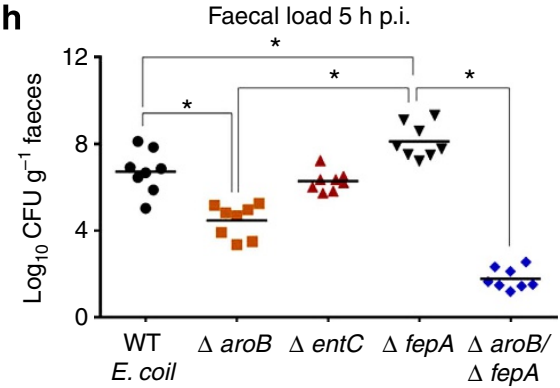

$\mathbf{f}$

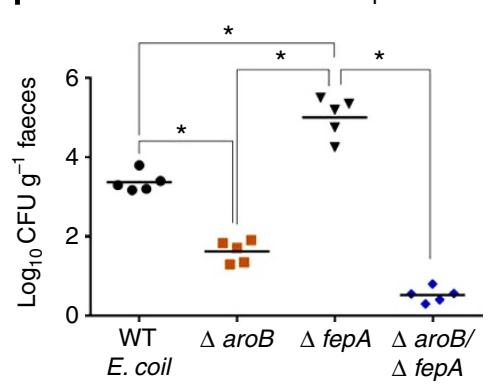

i

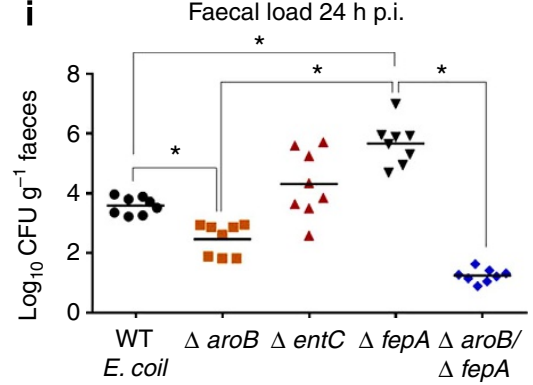

g

Caecal load 48 h p.i.

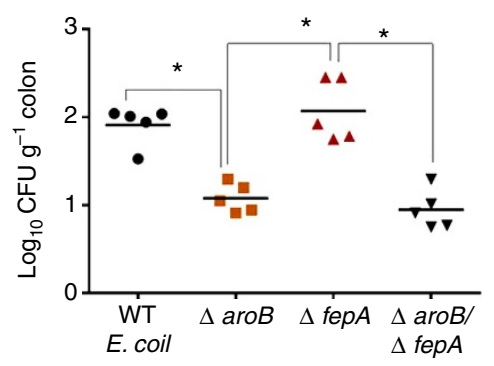

j

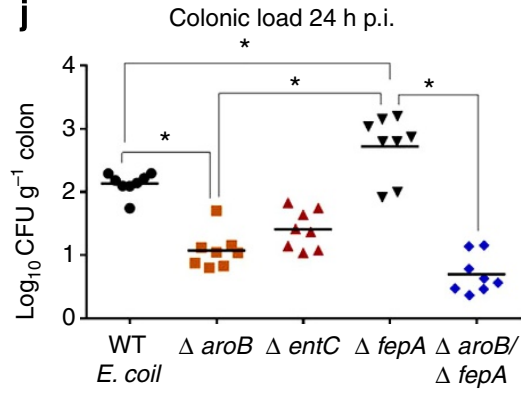

Colonic load 24 h p.i.

Figure 4 | Ent provides survival advantage to $\boldsymbol{E}$. coli in vitro and in the inflamed gut in vivo. Overnight-grown WT E. coli in LB medium was resuspended in PBS and then incubated with MPO $\left(5 \mu \mathrm{gl}^{-1}\right)$ and $\mathrm{H}_{2} \mathrm{O}_{2}(30 \mu \mathrm{M})$ with or without Ent (30 $\left.\mu \mathrm{M}\right)$ or DHBA $(300 \mu \mathrm{M})$ for 30 min. (a) Image displays overnightgrown bacterial colonies on an agar plate. (b) Bar graph representing \% survival of WT E. coli under different treatment. WT E. coli, $\triangle a r o B$ and $\triangle$ fepA mutants were grown in an iron-depleted medium for $6 \mathrm{~h}$ and subsequently examined for $\mathrm{MPO}-\mathrm{H}_{2} \mathrm{O}_{2}$-mediated bacterial killing as described above. (c) Bar graph represents \% survival of WT E. coli, $\Delta a r o B, \Delta f e p A$ and $\Delta a r o B / \Delta f e p A$ mutants. (d) Effect of $\Delta f e p A$ and $\Delta$ aroB $/ \Delta f e p A$ culture supernatant on survival of $\Delta$ aroB. $(\mathbf{e}-\mathbf{j})$ Streptomycin-pretreated Salmonella-induced gastroenteritis mice $(n=5)$ orally gavaged with WT E. coli, $\triangle \operatorname{aro} B, \Delta$ fepA or $\Delta$ aroB/ $\Delta$ fepA mutants $\left(1.0 \times 10^{8} \mathrm{CFU}\right.$ per mouse). Faecal CFUs were quantified on selective (kanamycin $\left.30 \mu \mathrm{g} \mathrm{ml}^{-1}\right)$ agar plates. Dot plot represents faecal shedding of $E$. coli mutants from the Salmonella-induced gastroenteritis model after (e) 5 and (f) $48 \mathrm{~h}$. (g) Caecal bacterial load at $48 \mathrm{~h}$. Mice $(n=8)$ exhibiting symptoms of DSS-induced colonic inflammation were orally gavaged with $10 \times 10^{9} \mathrm{CFU}$ of $\Delta$ aroB, $\Delta$ entC, $\Delta$ fepA or $\Delta$ aroB/ $\Delta$ fepA mutants. Faecal shedding and colonic tissue loads of $E$. coli mutants were selectively quantified on kanamycin $\left(30 \mu \mathrm{g} \mathrm{ml}^{-1}\right)$ agar plates. Dot plot represents faecal shedding of $E$. coli mutants from DSSinduced colitic mice after (h) $5 \mathrm{~h}$ and (i) $24 \mathrm{~h}$. (j) Colonic tissue load after $24 \mathrm{~h}$. Results are expressed as mean \pm s.e.m. and are representative of two independent mice experiments. In vitro experiments are representative of four independent experiments. ${ }^{\star} P<0.05$, unpaired $t$-test.

(Supplementary Fig. 8). To evaluate the effect of $\mathrm{MPO}-\mathrm{H}_{2} \mathrm{O}_{2}$ on killing bacteria that either lack or overproduce Ent, we exposed WT E. coli and the Ent mutants, $\triangle a r o B, \Delta f e p A$ and $\triangle a r o B / \Delta f e p A$, to $\mathrm{MPO}-\mathrm{H}_{2} \mathrm{O}_{2}$. The Ent-overproducing $E$. coli mutant $\Delta$ fepA was substantially protected from $\mathrm{MPO}-\mathrm{H}_{2} \mathrm{O}_{2}$-induced bacterial killing (Fig. 4c) compared with WT E. coli and the Ent- 
deficient $\triangle a r o B$ and $\triangle a r o B / \triangle f e p A$ mutants, which survived poorly (Fig. 4c). Moreover, supernatants from the $\triangle f e p A$ mutant grown in iron-depleted LB substantially rescued $\triangle a r o B$ from MPO- $\mathrm{H}_{2} \mathrm{O}_{2}$-induced killing (Fig. 4d), whereas supernatants from $\triangle a r o B / \Delta f e p A$ mutants offered no protection, suggesting that Ent released in the media from $\triangle$ fepA might protect $\triangle a r o B$ against MPO-mediated bacterial killing.

We further investigated the extent to which Ent-mediated inhibition of MPO activity protects bacteria in vivo and offers a survival advantage in the inflamed gut. On the basis of the in vitro studies, we hypothesized that the Ent-overproducing $\triangle f e p A$ mutant, but not the $\triangle a r o B, \Delta e n t C$ or $\triangle a r o B / \Delta f e p A$ mutants, would demonstrate an in vivo survival advantage in the inflamed gut. After oral gavage of bacteria to mice with robust caecal inflammation in Salmonella gastroenteritis and the DSS-induced colonic inflammation model, we monitored faecal shedding and tissue loads of mutant bacteria. We observed a significant increase in faecal shedding of $\triangle f e p A$ mutants relative to WT E. coli, $\Delta e n t C$, $\triangle a r o B$ and $\triangle a r o B / \Delta f e p A$ mutants (Fig. 4e,f,h,i), suggesting that Ent-null mutants were either killed while passing through the intestine or better colonized the inflamed intestinal tract. To distinguish between these two possibilities, we quantified caecal and colonic loads of these Ent mutants. The Ent-overproducing $\triangle$ fepA mutant superiorly colonized in the caecal (Fig. 4g) and colonic tissue (Fig. 4j) compared with the Ent mutants. Taken together, these results indicate that E. coli overproducing Ent survive and colonize better in inflamed colons than do mutants lacking Ent.

Lcn2 abrogates Ent-mediated inhibition of MPO activity. Having established that MPO secreted by neutrophil granules at the site of infection is vulnerable to inactivation by bacterial siderophores, we next sought to identify potential host proteins that may counter Ent-mediated MPO inactivation. Lcn2, a host siderophore-binding protein present in specific granules of neutrophils, is additionally produced by gut epithelial cells during inflammation ${ }^{27,29,30}$. To test the possibility that $\mathrm{Lcn} 2$ can counter Ent-mediated MPO inhibition, we performed an MPO assay in which Ent or DHBA was pre-incubated with recombinant human or mouse-Lcn2 (siderophore- and iron free). Notably, both human and mouse Lcn2-treated Ent and DHBA failed to inhibit MPO (Fig. 5a) and LPO activity (Fig. 5b). Together, these results suggest that MPO may be at risk for inactivation by bacterial siderophores upon discharge by neutrophils at the site of infection. However, Lcn2 can rescue MPO from bacterial siderophores, therefore restoring its bactericidal capabilities.

\section{Discussion}

Microbiotal dysbiosis is commonly associated with IBD and is characterized by a bloom in bacterial species belonging to phylum proteobacteria such as E. coli, a typically innocuous resident of the gastrointestinal tract. For instance, Enterobacteriaceae represent $86 \%$ of the total bacterial community in ileal Crohn's disease patients ${ }^{31}$. In this study, we demonstrate a novel survival mechanism in which siderophores expressed by E. coli can inhibit the host's potent neutrophilic antibacterial enzyme MPO in a dose-dependent fashion. Such inhibition of MPO requires iron-free Ent and DHBA. By spectral analysis, we provide evidence that Ent and DHBA divert the peroxidase from the halogenation cycle by acting as potent suicide substrates of the peroxidase-catalysed reaction. The E. coli mutant $(\triangle f e p A)$, which overproduces Ent, exhibits greater MPO inhibitory activity and colonizes the inflamed mouse intestine better than mutants that completely lack Ent expression ( $a r o B)$. The host's siderophorebinding protein, Lcn2, can inhibit Ent- and DHBA-mediated MPO inhibition, thereby neutralizing the E. coli survival advantage.

IBDs are characterized by an elevated innate immune response accompanied by generation of reactive oxygen species (ROS) and nitric oxide radicals. To survive in the chemically hostile environment, $E$. coli or the microbiota must be equipped with endogenous machinery or molecules that can effectively neutralize host-derived pro-oxidants. Accordingly, several studies have demonstrated that bacterial siderophores can act as ROSneutralizing agents ${ }^{32-34}$. Recently, Adler et al. demonstrated that an entE mutant strain impaired in Ent production was unable to form colonies on M9 agar medium; however, addition of Ent or ascorbate rescued the mutant. Their study also demonstrated that Ent-Fe ${ }^{+3}$ hydrolysis in the cell cytoplasm not only fulfills iron needs, but also provides the cell with a molecule rich in hydroxyl groups that can scavenge $\operatorname{ROS}^{35}$. The same group previously demonstrated that Ent is necessary to protect $E$. coli against pyochelin, a siderophore from $P$. aeruginosa-induced oxidative stress $^{36}$. Thus, in addition to helping E. coli acquire iron, Ent may also grant protection in an oxidizing environment in several ways. Being rich in $-\mathrm{OH}$ groups, Ent could neutralize external host- a

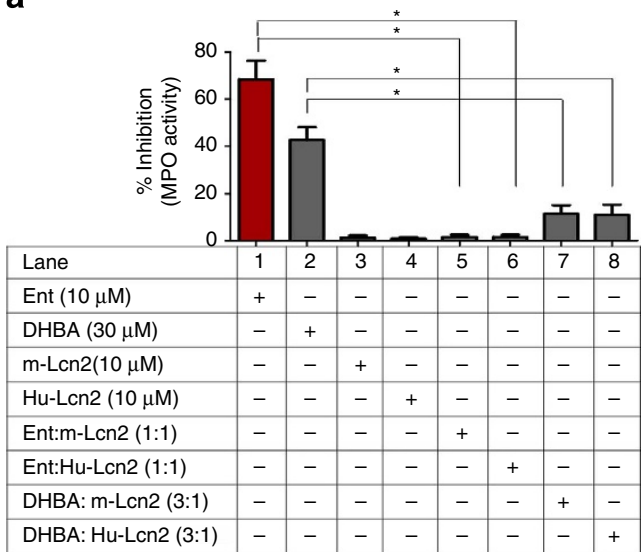

b

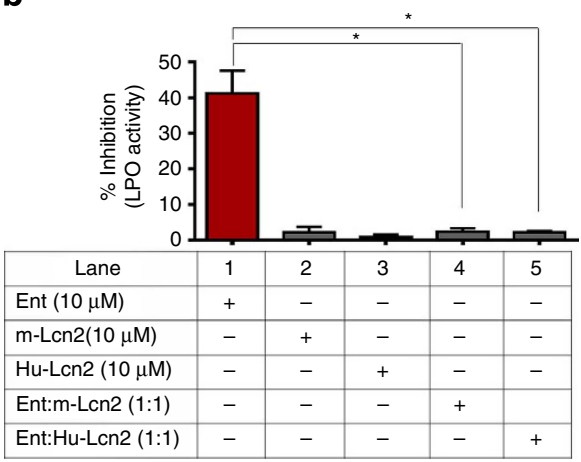

Figure 5 | Ent bound to Hu-Len2 or mouse-Len2 failed to inhibit MPO activity. Ent (10 $\mu \mathrm{M})$ or DHBA (30 $\mu \mathrm{M})$ was pre-incubated with or without $\mathrm{Hu}-\mathrm{Lcn} 2(10 \mu \mathrm{M})$ or mouse-Lcn2 $(10 \mu \mathrm{M})$ for 3 min and the resulting complex was subsequently incubated with MPO (100 mU) or LPO (50 $\left.\mu \mathrm{g} \mathrm{ml}{ }^{-1}\right)$ for 10 min. After binding to Hu-Lcn2 or mouse-Lcn2, Ent and DHBA failed to inhibit MPO (a) or LPO (b) activity. Results are expressed as mean \pm s.e.m. and are representative of three independent experiments. ${ }^{\star} P<0.05$, unpaired $t$-test. 
derived oxidative damage-inducing molecules. Further, Ent may also serve as an endogenous antioxidant to the bacterium when iron is internalized. Finally, Ent could reduce iron availability to immune cells, which require iron to produce hydroxyl radicals via the Haber-Weiss reaction. Our study supports the notion of Ent protecting against oxidative stress, but in a new dimension: through blocking the production of the potent oxidizing agent hypohalous acid. Notably, both salmochelin (glycosylated enterobactin) and non-catecholate siderophores (that is, yersiniabactin and ferrichrome) failed to inhibit MPO activity at concentrations even higher than Ent. This result suggests that MPO activity is specifically inhibited by Ent.

Neutrophils are the richest source of Lcn2 (ref. 37). It has been shown that mucosal Lcn2 exhibits pro-inflammatory and ironsequestering activity in response to Ent $^{38}$. Specifically, Ent-bound Lcn2 can activate mucosal epithelia to secrete interleukin-8, a potent neutrophil chemotactic agent. Thus, Lcn2 represents a novel mechanism of sensing microbial metabolites to modulate the host immune response. Accordingly, mice lacking Lcn2 are sensitive to $E$. coli infections ${ }^{39,40}$. Consistent with these results, our study indicates that the pro-inflammatory activity of Lcn2 could be also due to its ability to bind Ent and preserve MPO activity, ultimately resulting in a heightened inflammatory response mediated by hypohalous acid via generation of $\operatorname{ROS}^{41}$.

Acquiring iron from the host represents a major challenge for bacterial aerobic metabolism and growth. Bacteria overcome this limitation by producing siderophores, which have high affinity for iron. Further, inflammatory diseases, IBD in particular, are associated with a low iron environment or anaemia due to upregulation of hepcidin and/or increased expression of ironsequestering proteins ${ }^{42,43}$. As such, the iron-depleted environment may signal or impose selective pressures on E. coli to produce higher levels of Ent or modified siderophores. These siderophores not only help in acquiring iron but may also function as MPO inhibitors. Our study suggests that the host's innate immune protein Lcn2, which can bind Ent and prevent MPO inhibition, may exert immune pressure on $E$. coli to express altered and stealth siderophores. For example, E. coli is known to express altered stealth siderophores such as salmochelin and yersiniabactin, which display increased iron-scavenging activity, thereby favouring bacterial growth and limiting the availability of iron to immune cells $^{44}$. These stealth siderophores are known to avoid recognition by Lcn2, which may grant the bacteria superior survival in the inflamed environment. Crouch et al. ${ }^{45}$ showed that Salmonella synthesizes approximately equal amounts of enterobactin and salmochelin. On a molar ratio, Salmonella may have less enterobactin compared with $E$. coli and consequently exhibited reduced MPO inhibitory activity, because both enterobactin and salmochelin are products of the same pathway. Herein, we demonstrate that salmochelin fails to inhibit MPO activity. In addition, Salmonella and certain clinical isolates of E. coli are known to convert the cyclic enterobactin to linearized enterobactin ${ }^{46,47}$. However, it is unclear whether linearized enterobactin avoids Lcn2 recognition or displays altered MPO inhibitory activity. The flexibility of $E$. coli in expressing multiple and independent siderophores may explain its success as an opportunistic pathogen in extraintestinal tissues and in the evolution of several E. coli pathotypes of major public health concern $^{48}$.

Taken together, our study demonstrates that the E. coli siderophore Ent and its monomeric form DHBA inhibit MPO activity by acting as suicidal substrates, providing a distinct survival advantage to $E$. coli in the inflamed gut. Thus, E. coli siderophores serve as dual-purpose molecules, both in iron acquisition and in protection from oxidative stress imposed by host innate immunity. Further, the neutralization of Ent-mediated inhibition of MPO

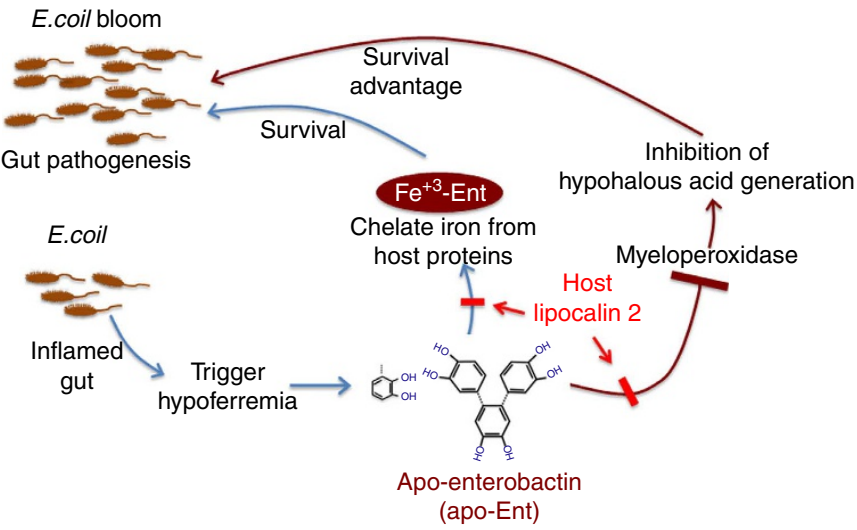

Figure 6 | Potential mechanism by which Ent-mediated MPO inhibition provides a survival advantage to $E$. coli in the inflamed intestine.

Hypoferremia in the inflamed gut signals bacteria ( $E$. coli) to produce more enterobactin (Ent), which not only helps to efficiently acquire iron from the environment but also inactivates host antibacterial enzyme

myeloperoxidase (MPO). The dual activities of Ent provide a survival advantage despite the inflammatory milieu, resulting in $E$. coli bloom. The inhibition of MPO activity by Ent is counter-regulated by host siderophorebinding protein, Lipocalin 2.

activity by Lcn 2 may exert selective immune pressure on $E$. coli to evolve and synthesize stealth siderophores (for example, aerobactin and yersiniabactin), which resist chelation by host Lcn2. Moreover, the interplay between siderophores and host innate immunity may result in the altered metagenomics, which could be a potential mechanism for the E. coli bloom in IBD. Our study also suggests that Ent may interfere with other $\mathrm{Fe}^{+3}$-dependent ROS-generating enzymes (for example, NADPH oxidases) ${ }^{49}$ and overall reduce the oxidative stress imposed by the host innate immunity. Future studies with MPO-deficient mice are warranted to convincingly demonstrate to what extent enterobactin-MPO interaction dictates E. coli survival. It is interesting to note that E. coli Nissle 1917, a member of the family Enterobacteriaceae, encodes four siderophores including Ent, salmochelin, aerobactin and yersiniabactin ${ }^{44}$. This strain, also known as 'Mutaflor', has been used in Germany for many years to treat colitis ${ }^{50}$ and constipation $^{51}$. In the same line, this study may facilitate further investigation of the utility of siderophore-based molecules to target $\mathrm{MPO}$, a culprit in the generation of deleterious oxidatively modified biomolecules, which play a critical role in multiple chronic diseases ${ }^{52}$.

In conclusion, as schematically represented in Fig. 6, we have demonstrated that siderophores generated by $E$. coli, by virtue of inhibiting the MPO-catalysed reaction with $\mathrm{H}_{2} \mathrm{O}_{2}$, define a new defence mechanism of microbiotal warfare against the host innate immune system.

\section{Methods}

Reagents. Human MPO, iron-free Ent, ferric chloride, hydrogen peroxide, $O$-dianisidine hydrochloride, DHBA, ABAH, dimethyl sulfoxide, ferrichrome and $2,2^{\prime}$ dipyridyl were procured from Sigma (St Louis, MO). Yersiniabactin (ybt) and salmochelin S4 were obtained from EMC microcollections ( $\mathrm{GmbH}$, Germany). Bovine milk LPO was purchased from Worthington Biochemical Corp., (Lakewood, NJ). Guaiacol (2-methoxyphenol) was obtained from Alfa Aesar (MA, USA) Human Lcn2 (also known as neutrophil gelatinase-associated lipocalin), murine Lcn2 (also known as 24p3) and human MPO were purchased from R\&D Systems (Minneapolis, MN). The commercially available recombinant $\mathrm{Lcn} 2$ proteins were free of endotoxin, siderophore and iron.

E. coli and its isogenic siderophore mutants. WT E. coli (EcK12) and its isogenic mutant of 3-dehydroquinate synthase $(\triangle a r o B)$ were obtained from The E. coli Genetic Stock Center, Yale University. WT and its isogenic isochorismate synthase 
$1(\Delta e n t C)$, ferrienterobactin permease $(\triangle$ fep $A)$ and aroB-fepA double mutants $(\triangle a r o B / \Delta f e p A)$ were gifts from Dr Kathleen Postle, Pennsylvania State University. The EcK12 strain has been shown to colonize poorly in the murine intestinal tract $^{53}$ and is incapable of disseminating to extraintestinal tissues, such as the spleen, liver and mesenteric lymph nodes ${ }^{54}$.

Bacterial cultures. Non-pathogenic WT E. coli and its isogenic Ent mutants $(\Delta e n t C, \triangle a r o B, \triangle f e p A$ and $\triangle a r o B / \Delta f e p A)$ were grown in LB media overnight at $37^{\circ} \mathrm{C} .2,2^{\prime}$-dipyridyl $(350 \mu \mathrm{M})$, which complexes with transition metals, was added to make the LB media iron deficient. The bacteria were pelleted and grown in LB media with or without $2,2^{\prime}$-dipyridyl for $6 \mathrm{~h}^{38}$. The bacterial CFUs were adjusted based on the optical density at $600 \mathrm{~nm}$. Media containing an equal number of bacteria were centrifuged and clear supernatants were used for the in vitro MPO and LPO activity assay.

Mice. Balb/c mice were bred and maintained at the animal house facility at the Pennsylvania State University. Animal experimental protocols were approved by the Pennsylvania State University IACUC committee.

MPO and LPO assay. Human MPO (50 $\mathrm{U} \mathrm{mg}^{-1}$ protein) was reconstituted with $0.1 \mathrm{M}$ potassium phosphate buffer $(\mathrm{pH} 6.0)$ and stored at $-80^{\circ} \mathrm{C}$. Aferric Ent was reconstituted in dimethyl sulfoxide and stored at $-80^{\circ} \mathrm{C}$. DHBA was reconstituted in PBS ( $\mathrm{pH}$ 8.0). Salmochelin was reconstituted in water and yersiniabactin was dissolved in methanol. Assays were carried out in 96-well plates (Corning) in triplicate with appropriate vehicles/buffers throughout the study. This method is based on the principle that MPO oxidizes guaiacol in the presence of $\mathrm{H}_{2} \mathrm{O}_{2}$ to chromophore 3,3'-dimethoxy-4, $4^{\prime}$-biphenylquinone ${ }^{55}$. Briefly, MPO $(100 \mathrm{mU}$, final concentration) or LPO $\left(50 \mu \mathrm{g} \mathrm{ml}^{-1}\right.$, final concentration) were pre-incubated with vehicle/appropriate buffers, various concentrations of siderophores or indicated molecules for $10 \mathrm{~min}$. The reaction was initiated by adding final concentrations of $100 \mathrm{mM}$ guaiacol and $6.7 \times 10^{-3} \% \mathrm{H}_{2} \mathrm{O}_{2}$. The change in absorbance at $470 \mathrm{~nm}$ was measured over a period of $20 \mathrm{~min}$ at 1-min intervals. One unit of MPO activity was defined as the amount that degraded $1.0 \mu \mathrm{mol}$ of peroxidase per minute at $25^{\circ} \mathrm{C}$. As a positive control, an MPO inhibitor, ABAH was used at half-maximal inhibitory concentration of $16 \mu \mathrm{M}$ in all of the experiments. We also used MPO oxidized by $\mathrm{O}$-dianisidine- $\mathrm{HCl}$ in the presence of $\mathrm{H}_{2} \mathrm{O}_{2}$ (ref. 56) and observed similar results. The data presented in this study employed the guaiacol method, as it was found to be more sensitive than $O$-dianisidine.

CAS assay for Ent-secreting E. coli mutants. CAS agar plates were prepared according to the protocol as outlined in ref. 57. Briefly, overnight bacterial cultures grown in LB media were placed on CAS agar plates, incubated overnight at $37^{\circ} \mathrm{C}$ and monitored for orange-coloured halo formation. CAS gives a distinctive blue colour when in complex with iron. When the iron is instead chelated by bacterial siderophores, orange halos develop around the siderophore-producing bacteria. The intensity of halo formation is directly proportional to the concentration of siderophores secreted by the bacteria. Purified Ent (Sigma) was used as a positive control.

In vitro bacterial killing assay. The in vitro bacterial killing assay was performed as described by Atosuo and Lilius ${ }^{58}$. Briefly, the MPO reaction mixture was prepared by first adding the MPO $\left(5.0 \mu \mathrm{g} \mathrm{ml}^{-1}, \mathrm{R} \& D\right.$ systems $)$ with or without Ent $(10$ or $30 \mu \mathrm{M})$ or DHBA $(300 \mu \mathrm{M})$ at final concentrations in sterile conditions. Hydrogen peroxide $(30 \mu \mathrm{M})$ was then added to the reaction mixture and incubated at room temperature for $5 \mathrm{~min}$. Finally, WT or Ent mutant bacteria grown overnight at $37^{\circ} \mathrm{C}$ with 250 r.p.m. shaking $\left(2 \times 10^{6} \mathrm{CFU} \mathrm{ml}^{-1}\right)$ were added to the reaction mixture. In some experiments, Ent $(10 \mu \mathrm{M})$ or DHBA $(30 \mu \mathrm{M})$ was incubated with $10 \mu \mathrm{M}$ of $\mathrm{Hu}-\mathrm{Lcn} 2$ or murine Lcn2 before being added to the reaction mixture. After $30 \mathrm{~min}$ of incubation at room temperature, the reaction mixture was serially diluted and plated on non-selective LB plates in triplicates. Plates were incubated overnight at $37^{\circ} \mathrm{C}$ and bacterial $\mathrm{CFU}$ on plates were quantified.

\section{Streptomycin-pretreated Salmonella-induced gastroenteritis. Eight-week-old} $\mathrm{Balb} / \mathrm{c}$ female mice were pretreated with streptomycin orally and subsequently infected with Salmonella to induce colitis as described previously ${ }^{27}$. On the day of the experiment, water and food were withdrawn $4 \mathrm{~h}$ before treatment and $20 \mathrm{mg}$ of streptomycin in $100 \mu \mathrm{l}$ of sterile water was administered by oral gavage. Afterwards, animals were supplied with water and food ad libitum. At $20 \mathrm{~h}$ after streptomycin treatment, water and food were withdrawn again for $4 \mathrm{~h}$ before the mice were orally administered $10^{8} \mathrm{CFU}$ per mouse of Salmonella enterica serovar Typhimurium (SL3201) followed by $10^{8} \mathrm{CFU}$ per mouse E. coli Ent mutants, and were subsequently euthanized at $48 \mathrm{~h}$. Thoroughly washed caeca (free from faecal matter) were used for the MPO activity assay as described above.
DSS-induced colitis in mice. Eight-week-old female Balb/c mice $(n=4-5)$ were administered 2.5\% DSS (MP Biomedicals) in drinking water over a period of 7 days ${ }^{59}$. Induction of colonic inflammation was confirmed via faecal occult blood, diarrhoea and loss in body weight. Mice were orally inoculated $\left(1 \times 10^{9} \mathrm{CFU}\right.$ per mouse) with WT E. coli or its isogenic Ent mutants $\triangle a r o B, \Delta e n t C$ (lacks Ent expression), $\Delta f e p A$ (overproduces Ent) or $\Delta a r o B / \Delta f e p A$ (lacks Ent expression). In another set of experiments, DSS-induced colitic mice were orally administered DHBA $\left(100 \mathrm{mg} \mathrm{kg}^{-1} \text { body weight in } 0.1 \mathrm{ml} \text { of } 5 \% \mathrm{NaHCO}_{3}\right)^{60}$. Five hours after administration of either bacteria or $3 \mathrm{~h}$ after DHBA, mice were euthanized via $\mathrm{CO}_{2}$ asphyxiation, and colons were harvested for the MPO activity assay. Briefly, colons were homogenized in $0.5 \%$ cetyltrimethyl ammonium bromide (Sigma) in potassium buffer ( $\mathrm{pH} 6.0$ ), freeze-thawed three times, sonicated and centrifuged. The MPO assay was performed in the clear supernatants as described above.

In vivo survival and colonization assay. Eight-week-old female $\mathrm{Balb} / \mathrm{c}$ mice $(n=4-5)$ were administered 2.5\% DSS (MP Biomedicals) in drinking water ${ }^{59}$. Induction of colonic inflammation was confirmed via faecal occult blood and loss in body weight. On day 5 of DSS treatment, mice were administered streptomycin ( $20 \mathrm{mg}$ per mouse) and after $24 \mathrm{~h}$, orally gavaged $\left(10 \times 10^{9} \mathrm{CFU}\right.$ per mouse) with either $\Delta a r o B$ or $\Delta e n t C$ (lacks Ent expression) or $\Delta f e p A$ (overproduces Ent) mutants. DSS was restricted to 5 days because mice treated with DSS for 7 days exhibited severe diarrhoea. Faecal shedding of bacteria was measured 5 and $24 \mathrm{~h}$ post inoculation using selective agar plates (kanamycin $30 \mu \mathrm{g} \mathrm{ml}^{-1}$ ). After $24 \mathrm{~h}$, mice were euthanized via $\mathrm{CO}_{2}$ asphyxiation. Colons were flushed gently with PBS to remove faecal matter, homogenized and used to quantitate the colonization of mutant bacteria on selective agar plates as described above.

Spectral analysis. Basal spectra were recorded at $412 \mathrm{~nm}$ for freshly reconstituted LPO $(2.6 \mathrm{nM})$ in phosphate buffer $(0.1 \mathrm{M})$. Spectral analysis of LPO during the oxidation of Ent, DHBA and ABAH was recorded at $412 \mathrm{~nm}$ using CARY50BIO UV-Visible Spectrophotometer. Briefly, LPO was incubated with either Ent, DHBA or $\mathrm{ABAH}(1,10$ or $30 \mu \mathrm{M}$, respectively) for $1 \mathrm{~min}$. The reaction was started by the addition of $30 \mu \mathrm{M} \mathrm{H}_{2} \mathrm{O}_{2}$. The spectra were recorded every $10 \mathrm{~s}$. Each spectrum is an average of three scans taken in $1.0 \mathrm{~s}^{24}$.

Histology and immunohistochemistry analysis. At termination of the experiment, caecum and colonic tissue were isolated and preserved in $10 \%$ buffered formalin solution for $24 \mathrm{~h}$. Caeca of all groups were processed for paraffin embedding and serial paraffin sections $(5 \mu \mathrm{m})$ were collected and stained with haematoxylin and eosin.

Colons obtained from different groups were frozen as Swiss roll in optimum cutting temperature (OCT, Tissue-Tek) compound for cryostat sectioning. Immunoreactivity to colonic macrophages was revealed using rat anti-mouse F4/80 antibody, clone Cl:A3-1 (1:50 dilution, AbD Serotec) as described earlier ${ }^{61}$. An appropriate negative isotype control (rat IgG2b) was included to omit nonspecific staining.

Statistical analysis. All experimental results were reproduced in at least three independent experiments performed in triplicate. Results are expressed as means with their s.e. Significance of difference between groups was analysed by an unpaired $t$-test using GraphPad Prism software 6.0. A $P$ value less than 0.05 was considered statistically significant.

\section{References}

1. Goetz, D. H. et al. The neutrophil lipocalin NGAL is a bacteriostatic agent that interferes with siderophore-mediated iron acquisition. Mol. Cell 10, 1033-1043 (2002).

2. Nairz, M., Schroll, A., Sonnweber, T. \& Weiss, G. The struggle for iron-a metal at the host-pathogen interface. Cell Microbiol. 12, 1691-1702 (2010).

3. Skaar, E. P. The battle for iron between bacterial pathogens and their vertebrate hosts. PLoS Pathog. 6, e1000949 (2010).

4. Lin, H., Fischbach, M. A., Liu, D. R. \& Walsh, C. T. In vitro characterization of salmochelin and enterobactin trilactone hydrolases IroD, IroE, and Fes. J. Am. Chem. Soc. 127, 11075-11084 (2005).

5. Bellaire, B. H., Elzer, P. H., Baldwin, C. L. \& Roop, 2nd R. M. Production of the siderophore 2,3-dihydroxybenzoic acid is required for wild-type growth of Brucella abortus in the presence of erythritol under low-iron conditions in vitro. Infect. Immun. 71, 2927-2832 (2003).

6. Williams, P. H. Novel iron uptake system specified by ColV plasmids: an important component in the virulence of invasive strains of Escherichia coli. Infect. Immun. 26, 925-932 (1979).

7. Lawlor, M. S., O'Connor, C. \& Miller, V. L. Yersiniabactin is a virulence factor for Klebsiella pneumoniae during pulmonary infection. Infect. Immun. 75, 1463-1472 (2007).

8. Ratledge, C. \& Dover, L. G. Iron metabolism in pathogenic bacteria. Annu. Rev. Microbiol. 54, 881-941 (2000). 
9. Cusumano, C. K., Hung, C. S., Chen, S. L. \& Hultgren, S. J. Virulence plasmid harbored by uropathogenic Escherichia coli functions in acute stages of pathogenesis. Infect. Immun. 78, 1457-1467 (2010).

10. Klebanoff, S. J., Kettle, A. J., Rosen, H., Winterbourn, C. C. \& Nauseef, W. M. Myeloperoxidase: a front-line defender against phagocytosed microorganisms. J. Leukoc. Biol. 93, 185-198 (2013).

11. Sharma, S. et al. Lactoperoxidase: structural insights into the function,ligand binding and inhibition. Int. J. Biochem. Mol. Biol. 4, 108-128 (2013).

12. Chakraborty, S., Kaur, S., Guha, S. \& Batra, S. K. The multifaceted roles of neutrophil gelatinase associated lipocalin (NGAL) in inflammation and cancer. Biochim. Biophys. Acta 1826, 129-169 (2012).

13. Vujkovic-Cvijin, I. et al. Dysbiosis of the gut microbiota is associated with hiv disease progression and tryptophan catabolism. Sci. Transl. Med. 5, 193 ra191 (2013).

14. Normann, E., Fahlen, A., Engstrand, L. \& Lilja, H. E. Intestinal microbial profiles in extremely preterm infants with and without necrotizing enterocolitis. Acta Paediatr. 102, 129-136 (2013).

15. Baumgart, M. et al. Culture independent analysis of ileal mucosa reveals a selective increase in invasive Escherichia coli of novel phylogeny relative to depletion of Clostridiales in Crohn's disease involving the ileum. ISME J. 1, 403-418 (2007).

16. Carroll, I. M., Ringel-Kulka, T., Siddle, J. P. \& Ringel, Y. Alterations in composition and diversity of the intestinal microbiota in patients with diarrheapredominant irritable bowel syndrome. Neurogastroenterol. Motil. 24, 521-530 e248 (2012).

17. Hildebrand, F. et al. Inflammation-associated enterotypes, host genotype, cage and inter-individual effects drive gut microbiota variation in common laboratory mice. Genome Biol. 14, R4 (2013).

18. Spees, A. M. et al. Streptomycin-induced inflammation enhances Escherichia coli gut colonization through nitrate respiration. mBio 4, e00430-13 (2013).

19. Lupp, C. et al. Host-mediated inflammation disrupts the intestinal microbiota and promotes the overgrowth of Enterobacteriaceae. Cell Host Microbe 2, 204 (2007).

20. Raetz, M. et al. Parasite-induced TH1 cells and intestinal dysbiosis cooperate in IFN-gamma-dependent elimination of Paneth cells. Nat. Immunol. 14, 136-142 (2013).

21. Winter, S. E. \& Baumler, A. J. Why related bacterial species bloom simultaneously in the gut: principles underlying the 'Like will to like' concept. Cell Microbiol. 16, 179-184 (2013).

22. Winter, S. E., Lopez, C. A. \& Baumler, A. J. The dynamics of gut-associated microbial communities during inflammation. EMBO Rep. 14, 319-327 (2013).

23. Winter, S. E. et al. Host-derived nitrate boosts growth of E. coli in the inflamed gut. Science 339, 708-711 (2013).

24. Kettle, A. J., Gedye, C. A. \& Winterbourn, C. C. Mechanism of inactivation of myeloperoxidase by 4-aminobenzoic acid hydrazide. Biochem. J. 321, 503-508 (1997).

25. Nemeth, E. et al. IL-6 mediates hypoferremia of inflammation by inducing the synthesis of the iron regulatory hormone hepcidin. J. Clin. Invest. 113, 1271-1276 (2004).

26. Darfeuille-Michaud, A. et al. Presence of adherent Escherichia coli strains in ileal mucosa of patients with Crohn's disease. Gastroenterology 115, 1405-1413 (1998).

27. Vijay-Kumar, M. et al. Flagellin suppresses epithelial apoptosis and limits disease during enteric infection. Am. J. Pathol. 169, 1686-1700 (2006).

28. Vijay-Kumar, M. et al. Toll-like receptor 5-deficient mice have dysregulated intestinal gene expression and nonspecific resistance to Salmonella-induced typhoid-like disease. Infect. Immun. 76, 1276-1281 (2008).

29. Cowland, J. B., Sorensen, O. E., Sehested, M. \& Borregaard, N. Neutrophil gelatinase-associated lipocalin is up-regulated in human epithelial cells by IL-1 beta, but not by TNF-alpha. J. Immunol. 171, 6630-6639 (2003).

30. Vijay-Kumar, M. et al. Protein kinase R mediates intestinal epithelial gene remodeling in response to double-stranded RNA and live rotavirus. J. Immunol. 174, 6322-6331 (2005).

31. Willing, B. et al. Twin studies reveal specific imbalances in the mucosaassociated microbiota of patients with ileal Crohn's disease. Inflamm. Bowel Dis. 15, 653-660 (2009)

32. Reeder, B. J. \& Wilson, M. T. Desferrioxamine inhibits production of cytotoxic heme to protein cross-linked myoglobin: a mechanism to protect against oxidative stress without iron chelation. Chem. Res. Toxicol. 18, 1004-1011 (2005).

33. Achard, M. E. et al. An antioxidant role for catecholate siderophores in Salmonella. Biochem. J. 454, 543-549 (2013).

34. Paauw, A., Leverstein-van Hall, M. A., van Kessel, K. P., Verhoef, J. \& Fluit, A. C. Yersiniabactin reduces the respiratory oxidative stress response of innate immune cells. PLoS ONE 4, e8240 (2009).

35. Adler, C. et al. The alternative role of enterobactin as an oxidative stress protector allows Escherichia coli colony development. PLoS ONE 9, e84734 (2014).

36. Adler, C. et al. Catecholate siderophores protect bacteria from pyochelin toxicity. PLOS ONE 7, e46754 (2012).
37. Borregaard, N. \& Cowland, J. B. Neutrophil gelatinase-associated lipocalin, a siderophore-binding eukaryotic protein. Biometals 19, 211-215 (2006).

38. Bachman, M. A., Miller, V. L. \& Weiser, J. N. Mucosal lipocalin 2 has pro-inflammatory and iron-sequestering effects in response to bacterial enterobactin. PLoS Pathog. 5, e1000622 (2009).

39. Flo, T. H. et al. Lipocalin 2 mediates an innate immune response to bacterial infection by sequestrating iron. Nature 432, 917-921 (2004).

40. Berger, T. et al. Lipocalin 2-deficient mice exhibit increased sensitivity to Escherichia coli infection but not to ischemia-reperfusion injury. Proc. Nat Acad. Sci. USA. 103, 1834-1839 (2006).

41. Raffatellu, M. et al. Lipocalin-2 resistance confers an advantage to Salmonella enterica serotype Typhimurium for growth and survival in the inflamed intestine. Cell Host Microbe 5, 476-486 (2009).

42. Gisbert, J. P. \& Gomollon, F. Common misconceptions in the diagnosis and management of anemia in inflammatory bowel disease. Am. J. Gastroenterol. 103, 1299-1307 (2008)

43. Ganz, T. \& Nemeth, E. Hepcidin and iron homeostasis. Biochim. Biophys. Acto 1823, 1434-1443 (2012).

44. Faber, F. \& Baumler, A. J. The impact of intestinal inflammation on the nutritional environment of the gut microbiota. Immunol. Lett. 162, 48-53 (2014).

45. Crouch, M. L., Castor, M., Karlinsey, J. E., Kalhorn, T. \& Fang, F. C. Biosynthesis and IroC-dependent export of the siderophore salmochelin are essential for virulence of Salmonella enterica serovar Typhimurium. Mol. Microbiol. 67, 971-983 (2008).

46. Henderson, J. P. et al. Quantitative metabolomics reveals an epigenetic blueprint for iron acquisition in uropathogenic Escherichia coli. PLoS Pathog. 5, e1000305 (2009)

47. Fischbach, M. A., Lin, H., Liu, D. R. \& Walsh, C. T. How pathogenic bacteria evade mammalian sabotage in the battle for iron. Nat. Chem. Biol. 2, 132-138 (2006).

48. Croxen, M. A. et al. Recent advances in understanding enteric pathogenic Escherichia coli. Clin. Microbiol. Rev. 26, 822-880 (2013).

49. Lambeth, J. D. NOX enzymes and the biology of reactive oxygen. Nat. Rev. Immunol. 4, 181-189 (2004).

50. Schutz, E. [The treatment of intestinal diseases with Mutaflor. A multicenter retrospective study]. Fortschr. Med. 107, 599-602 (1989).

51. Mollenbrink, M. \& Bruckschen, E. [Treatment of chronic constipation with physiologic Escherichia coli bacteria. Results of a clinical study of the effectiveness and tolerance of microbiological therapy with the E. coli Nissle 1917 strain (Mutaflor)]. Med. Klin. 89, 587-593 (1994).

52. Sokolov, A. V. et al. Proatherogenic modification of LDL by surface-bound myeloperoxidase. Chem. Phys. Lipids 180, 72-80 (2014).

53. Heimesaat, M. M. et al. Colonization resistance against genetically modified Escherichia coli K12 (W3110) strains is abrogated following broad-spectrum antibiotic treatment and acute ileitis. Eur. J. Microbiol. Immunol. 3, 222-228 (2013).

54. Bereswill, S. et al. Pro-inflammatory potential of Escherichia coli strains K12 and Nissle 1917 in a murine model of acute ileitis. Eur. J. Microbiol. Immunol. 3, 126-134 (2013).

55. Capeillere-Blandin, C. Oxidation of guaiacol by myeloperoxidase: a two-electron-oxidized guaiacol transient species as a mediator of NADPH oxidation. Biochem. J. 336, 395-404 (1998).

56. Castaneda, F. E. et al. Targeted deletion of metalloproteinase 9 attenuates experimental colitis in mice: central role of epithelial-derived MMP. Gastroenterology 129, 1991-2008 (2005).

57. Schwyn, B. \& Neilands, J. B. Universal chemical assay for the detection and determination of siderophores. Anal. Biochem. 160, 47-56 (1987).

58. Atosuo, J. T. \& Lilius, E. M. The real-time-based assessment of the microbial killing by the antimicrobial compounds of neutrophils. ScientificWorldJournal 11, 2382-2390 (2011).

59. Chassaing, B., Aitken, J. D., Malleshappa, M. \& Vijay-Kumar, M. Dextran sulfate sodium (DSS)-induced colitis in mice. Curr. Protoc. Immunol. 104, Unit 1525 (2014; edited by John E. Coligan et al..

60. Graziano, J. H., Grady, R. W. \& Cerami, A. The identification of 2, 3-dihydroxybenzoic acid as a potentially useful iron-chelating drug. J. Pharmacol. Exp. Ther. 190, 570-575 (1974).

61. Khanna, V. et al. Cholesterol diet withdrawal leads to an initial plaque instability and subsequent regression of accelerated iliac artery atherosclerosis in rabbits. PLoS ONE 8, e77037 (2013).

\section{Acknowledgements}

This work was supported by grants from the National Institutes of Health K01 (DK083275), R03 (DK094864) and R01 (DK097865) to M.V.-K. We thank Dr Jim Imlay for providing E. coli mutants and critical input. We thank Dr Chobi Debroy, Director, E. coli Reference Center, for providing the E. coli LF82 strain. We acknowledge scientific and technical inputs given by Drs B.J. Cherayil (Harvard Medical School, Boston, MA), R.K. Strong (Fred Hutchinson Cancer Research Center, Seattle, WA) and J.P. Henderson 
(University of Washington, St Louis, MO). We greatly appreciate Drs Kathleen Postle for providing E. coli and their isogenic mutants, Tim Yosca for spectral analysis and Neela Yennawar, Department of Biochemistry and Molecular Biology, Pennsylvania State University, for advice and helpful discussions.

\section{Author contributions}

V.S. designed and performed the experiments, analysed the data and co-wrote the paper. B.S.Y. designed and performed experiments with bacterial mutants. X.X. performed animal experiments and histologic analysis. M.K. performed animal experiments. M.B., N.B. and B.J. contributed to the experimental design and analysis. M.V.-K. designed experiments, analysed the data and co-wrote the paper.

\section{Additional information}

Supplementary Information accompanies this paper at http://www.nature.com/ naturecommunications

Competing financial interests: The authors declare no competing financial interests.

Reprints and permission information is available online at http://npg.nature.com/ reprintsandpermissions/

How to cite this article: Singh, V. et al. Interplay between enterobactin, myeloperoxidase and lipocalin 2 regulates E. coli survival in the inflamed gut. Nat. Commun. 6:7113 doi: 10.1038/ncomms8113 (2015). 